\title{
Superinfection exclusion and the long-term survival of honey bees in Varroa-infested colonies
}

\author{
Gideon J Mordecai ${ }^{1,2}$, Laura E Brettell ${ }^{3}$, Stephen J Martin³, David Dixon ${ }^{1}$, Ian M Jones ${ }^{2}$ \\ and Declan C Schroeder ${ }^{1}$ \\ ${ }^{1}$ Viral Ecology, Marine Biological Association, Plymouth, UK; ${ }^{2}$ School of Biological Sciences, \\ University of Reading, Reading, UK and ${ }^{3}$ School of Environment and Life Sciences, The University of Salford, \\ Manchester, UK
}

\begin{abstract}
Over the past $\mathbf{5 0}$ years, many millions of European honey bee (Apis mellifera) colonies have died as the ectoparasitic mite, Varroa destructor, has spread around the world. Subsequent studies have indicated that the mite's association with a group of RNA viral pathogens (Deformed Wing Virus, DWV) correlates with colony death. Here, we propose a phenomenon known as superinfection exclusion that provides an explanation of how certain $A$. mellifera populations have survived, despite Varroa infestation and high DWV loads. Next-generation sequencing has shown that a non-lethal DWV variant 'type B' has become established in these colonies and that the lethal 'type A' DWV variant fails to persist in the bee population. We propose that this novel stable host-pathogen relationship prevents the accumulation of lethal variants, suggesting that this interaction could be exploited for the development of an effective treatment that minimises colony losses in the future.

The ISME Journal (2016) 10, 1182-1191; doi:10.1038/ismej.2015.186; published online 27 October 2015
\end{abstract}

\section{Introduction}

The recent global decline of the European honey bee (Apis mellifera) populations (Ratnieks and Carreck, 2010; Schroeder and Martin, 2012) is of grave concern because of their role as pollinators which contribute an estimated $\$ 225$ billion to the global economy (Gallai et al., 2009). For over half a century, the global spread of the ectoparasitic mite, Varroa destructor, has resulted in the death of many millions of managed and feral honey bee colonies (Martin et al., 2012; Schroeder and Martin, 2012; Thompson et al., 2014). The mite has introduced a new viral transmission route that has dramatically altered the viral landscape (Martin et al., 2012). This has resulted in a massive loss of diversity in Deformed Wing Virus (DWV) (Martin et al., 2012), the pathogen now linked with the collapse of honey bee colonies (Highfield et al., 2009; Di Prisco et al., 2011). However, prior to Varroa spread, DWV stably co-existed with honey bees (Martin et al., 2012) albeit at viral loads many orders of magnitude lower than is now observed (Martin et al., 2012; Mondet et al., 2014). For example, the recent arrival of Varroa into the Hawaiian honey bee population was accompanied by a million fold increase in the

Correspondence: D Schroeder, Viral Ecology, Marine Biological Association, Citadel Hill, Plymouth PL1 2PB, UK.

E-mail: dsch@mba.ac.uk

Received 9 July 2015; revised 25 August 2015; accepted 11 September 2015; published online 27 October 2015 viral load of DWV, loss of DWV diversity and the predominance of a single highly virulent DWV variant (type A) (Martin et al., 2012). These landscape scale changes have also been demonstrated at the individual honey bee level within the UK honey bee population. For example, Ryabov et al. (2014) demonstrated the dominance of a single variant of DWV when a mixture of viral strains were injected into developing pupae leading to a rapid loss of DWV diversity and million fold increase in viral loads.

DWV is a rapidly evolving group of closely related variants (de Miranda and Genersch, 2010), commonly referred to as a quasispecies (Domingo and Holland, 1997; Lauring and Andino, 2010). Within the DWV quasispecies, there are several master variants, each with its own swarm of variants. Each variant can form potential recombinants with other variants, within a swarm and between master variants. Kakugo virus is a variant of the DWV type A that differs from the master sequence (Lanzi et al., $2006)$ by $6 \%$ in the non-structural coding region (Fujiyuki et al., 2006; Baker and Schroeder, 2008), whereas Varroa destructor Virus-1 (VDV-1) (Ongus et al., 2004) is genetically dissimilar to DWV type A (84\% genome identity) and is referred to as DWV type B (Martin et al., 2012). Notably, both DWV type A and B master variants are able to replicate within mites and honey bees, and both have been detected in honey bees in the absence of Varroa (Yue and Genersch, 2005; Zioni et al., 2011; Martin et al., 2012). Recombinants between the variants have been 
reported (Moore et al., 2011; Zioni et al., 2011; Ryabov et al., 2014) and a novel recombinant between DWV type $\mathrm{A}$ and a new DWV master variant, type $\mathrm{C}$, has also been recently discovered (Mordecai et al., 2015), suggesting that they are part of the same quasispecies and share a recent common ancestor. DWV type A has been detected in honey bee populations around the world and in the presence of Varroa leads to colony death (Di Prisco et al., 2011; Martin et al., 2012), whereas there are no known instances of type B being linked to colony death. The role of the new type $C$ in overwintering colony losses is currently unclear (Highfield et al., 2009; Mordecai et al., 2015).

In the early 1990s, Varroa swept across the UK and was followed by widespread colony deaths 1-3 years later. To ensure the long-term survival of their honey bee colonies, beekeepers in Varroa-infested countries manage Varroa populations (Sumpter and Martin, 2004), largely through chemical methods. Nonetheless, there are reports of rare isolated untreated A. mellifera colonies of European origin thriving despite Varroa infestation, including cases on an island in Brazil (DeJong and Soares, 1997) and in small forest patches in France (Conte et al., 2007) and New York, USA (Seeley, 2007). The survival of these colonies is well documented and not questioned, however, the mechanism by which tolerance to Varroa and its association with DWV is maintained remains elusive. In the UK, a small number of beekeepers opted not to control their mite populations and, in most cases, lost their bees. However, one UK beekeeper, Ron Hoskins, initiated a closed breeding programme from colonies that survived the initial Varroa infestation and this isolated population of up to 40 colonies persists in Swindon, central England, without chemical control of Varroa (http://www.swindonhoneybeeconservation.org.uk/). The aim of this study was to assess the viral landscape in this apiary thereby determining whether the colonies remained disease-free owing to an absence of DWV. We show here that the Swindon apiary is dominated by an avirulent DWV type B master variant with the concomitant absence of the virulent DWV type A master variant. Taken together, these data suggest that a phenomenon known as superinfection exclusion (SIE) (Salaman, 1933; Labrie et al., 2010) is a plausible explanation for why this isolated UK honey bee population has survived, despite Varroa infestation and high DWV loads.

\section{Material and methods}

\section{Sample collection}

Pooled asymptomatic honey bees were collected from sites in Hawaii and from the Swindon Apiary. A time series of three hives in Swindon was taken (10 time points over a year, 4 of which were used for Illumina sequencing per hive (Illumina, Inc.,
San Diego, CA, USA)). Varroa samples were collected from the same three hives in the Swindon apiary alongside the honey bee samples.

\section{DWV detection assay}

RNA extractions, RT-PCR and High Resolution Melt (HRM) analysis were carried out according to a previous study (Martin et al., 2012). In brief, total RNA was extracted from whole worker honey bees using an RNeasy mini kit (Qiagen, Hilden, Germany), according to the manufacturer's instructions. RTPCR via oligo dT priming using previously designed DWV primers (Highfield et al., 2009) and subsequent HRM was carried out using Sensifast No-Rox One Step Kit (Bioline, London, UK). RNA was diluted to $\sim 100 \mathrm{ng} \mathrm{ll}^{-1}$ and $1 \mu \mathrm{l}$ of template RNA was added per reaction. The DWV load per worker honey bee was calculated according to the method developed by Highfield et al. (2009). The amount of RNA used for each RT-PCR reaction was normalised per bee and the DWV load per bee was calculated through a DWV cRNA standard curve conversion $(\mathrm{y}=-3.695 \mathrm{x}$ $+32.744)$.

\section{Bioinformatics pipeline}

Illumina Hi-seq $(2 \times 100 \mathrm{bp})$ pair-end sequencing was carried out by The Genome Analysis Centre (TGAC) and the University of Exeter. Total RNA was sequenced after a cDNA synthesis step with no amplification step necessary. Varroa RNA was pooled for three of the time points (January, April and May) prior to Illumina sequencing. A bioinformatics pipeline (Figure 1) was developed to accommodate the large amount of variation found within the DWV species complex. Firstly, the quality of the raw reads was verified using FastQC (Babraham Bioinformatics, Cambridge, UK). Samples were then converted from fastq to fasta using the fastq_to_fasta script which is part of the FASTX-toolkit (Hannonlab, http://hannonlab.cshl.edu/fastx_toolkit/). To isolate the reads sequenced from the DWV complex from the host and other contaminating sequences the BLASTn tool was used (Altschul et al., 1990). The reads were searched against a custom BLAST database containing the DWV, VDV-1 and Kakugo virus genomes, with an e value of 10e-05. BLAST was carried out against Read 1 of the Illumina data. The ncbiblastparser perl script (http://www.bioinfor matics-made-simple.com/2012/07/ncbi-blast-parserextract-query-and.html) was then used to parse and read the top hit of the BLAST output. Next, 'sed' and 'awk' scripts were used to delete empty lines and the reads, which contained 'nohits'. The corresponding BLAST hits were then pulled out from the Read 2 raw reads using QIIME. The paired reads were then balanced using a custom script written in $\mathrm{R}$ version 3.2.0 (R Core Team, 2015), which deletes any read in the Read 1 BLAST top hit file that did not have a pair in the corresponding read 2 BLAST top hit file and 


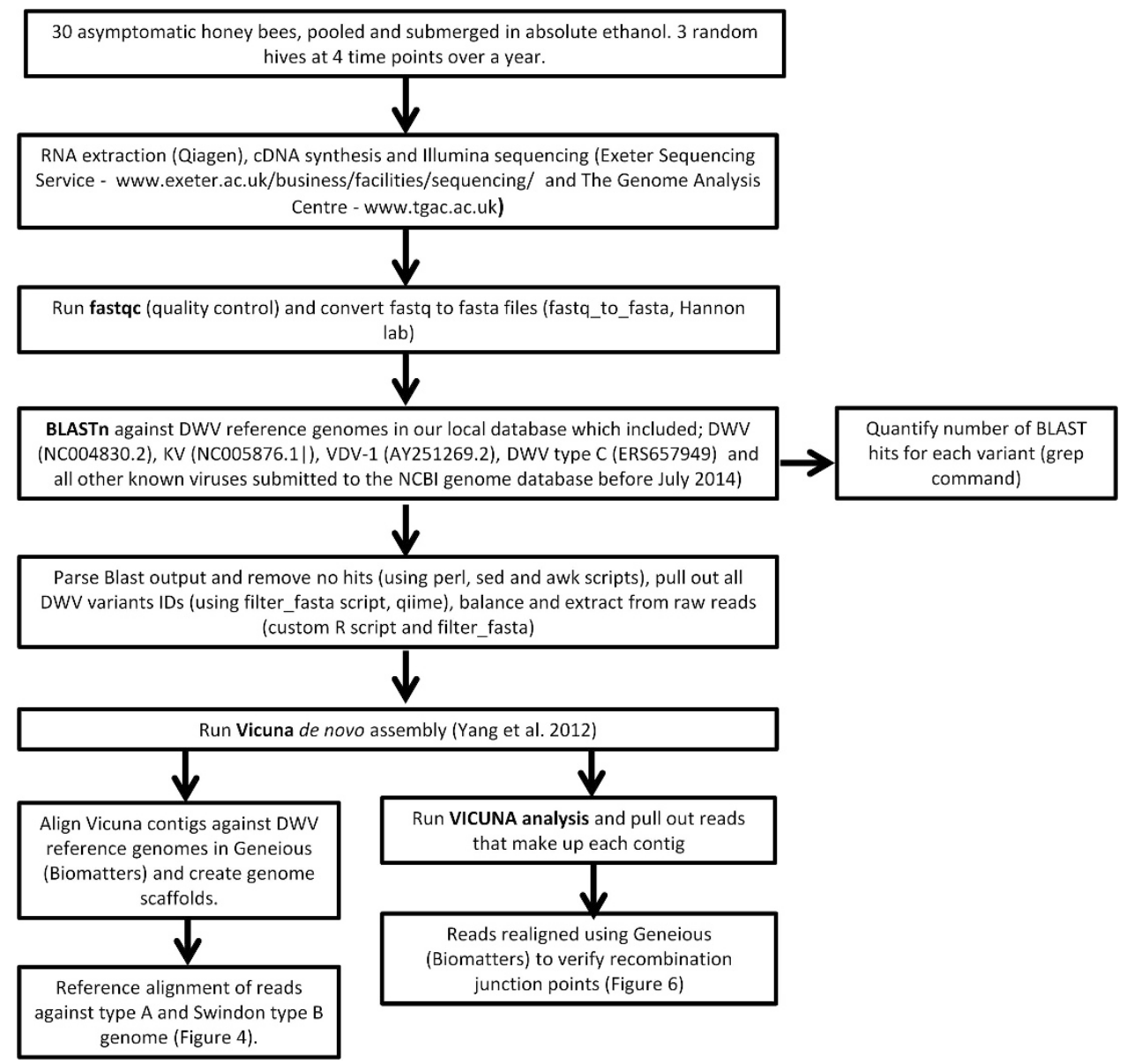

Figure 1 Bioinformatics pipeline leading to the application of the VICUNA de novo assembler.

vice versa. The balanced DWV family reads were then assembled using the VICUNA assembler, which was developed to generate consensus assemblies from genetically heterogeneous populations, specifically RNA viruses (Yang et al., 2012).

VICUNA contigs greater than $300 \mathrm{bp}$ in length were imported into Geneious (Version 7.04, created by Biomatters) and the 'Map to Reference tool' was used to align the contigs with the type A and B reference genomes. For several of the samples, the VICUNA assembly yielded full-length contigs that covered the whole genome, whereas others yielded only several smaller contigs (Supplementary Table S1). The ends of the contigs were then edited to remove discernable assembly or sequencing artefacts. Assembled DWV contigs were uploaded to the European Nucleotide Archive under the Study accession PRJEB8112. VICUNA contigs from hive 6 January 2013 were used to assemble a type B variant genome (accession number ERS754547).

The identity of the type B genome was compared with the VDV reference genome using the mVista tool (Supplementary Figure S1) (Frazer et al., 2004), and the phylogeny of the Swindon variant was determined from a neighbour-joining tree of the polyprotein encoding region of the DWV genome (Lanzi et al., 2006). In addition, genome scaffolding was carried out to produce full-length genomes representing the unique recombinant present in Swindon. SimPlot software was used to visualise the recombination event (Lole et al., 1999).

To investigate the genome coverage of each DWV variant in Swindon, reads were grouped per hive (Varroa samples were all grouped together) and competitively aligned against the type A reference genome (NC004830.2) and the Swindon type B genome using the Geneious map to reference tool. The maximum percentage of mismatches per read accepted was 5\% and no gaps per read were allowed.

To examine individual reads that make up the consensus sequence of each contig, the VICUNA analysis tool was used. To view the reads in Geneious, the VICUNA analysis output was modified by using a sed script to keep just the sequence reads. These were then converted from a tabular format into a fasta format using the python script 'tab2fasta.py' and then visualised using Geneious. To quantify the number of reads with sequences similar to either DWV variant (type A or B), the Illumina reads were searched against a viral database using BLAST and the number of top hits attributed to each reference genome was quantified. Finally, genome coverage was calculated using the Lander/Waterman equation (read length $\times$ number of reads/genome length), 
which estimates the depth of sequencing across the genome (Sims et al., 2014).

\section{Results and Discussion}

Using a combination of RT-qPCR, HRM (Martin et al., 2012) and Illumina $(2 \times 100 \mathrm{bp})$ Hi-seq sequencing (Figure 1), we investigated the DWV viral load and diversity in this small honey bee population in Swindon and their associated Varroa mites. Three hives were chosen at random and pools of 30 asymptomatic worker bees were sampled from inside the colony on 10 occasions at roughly monthly intervals between October 2012 and October 2013. RT-qPCR on an RNA-dependent RNA polymerase $(R d R p)$ gene fragment for all 30 samples collected confirmed the persistence of high DWV loads $\left(10^{7}\right.$ to $10^{8}$ copies per bee) during the entire study period in all three hives (Supplementary Table S2). Both the DWV load and prevalence found within this study suggest that DWV presence alone cannot explain colony losses as proposed in previous Hawaiian (Martin et al., 2012) and Devon, UK, studies (Highfield et al., 2009).

To explore other factors that might contribute to this discovery, we exploited the known nucleotide polymorphisms in the $R d R p$ gene fragment among the known DWV master variants (A, B and C) (Martin et al., 2012; Mordecai et al., 2015). HRM indicated the dominance of the type $B$ or $C$ master variant (Figure 2a), as these have similar melting temperatures (Martin et al., 2012; Mordecai et al., 2015). Only a single honey bee sample out of 30 tested contained both DWV type A and B/C, suggesting that although a colony can be exposed to type A, it fails to establish and neither persists nor accumulates. In contrast to the bee samples, the Varroa samples contained a greater mix of both DWV type A and $\mathrm{B} / \mathrm{C}$ (Figure 2b), although type $\mathrm{B} / \mathrm{C}$ remained the most prevalent. This prevalence of type B/C over A contrasts to what a previous study showed in Hawaii where the type A master variant dominated (Martin et al., 2012) and suggests that type B/C may be an avirulent form of DWV. However, given that HRM analysis only detects limited genomic change (within the RdRp gene fragment), the possibility of recombination outside the RdRp region cannot be excluded. Both Mordecai et al. (2015) and Ryabov et al. (2014) showed that certain recombinants of the master variants $\mathrm{A}-\mathrm{C}$ and $\mathrm{A}-\mathrm{B}$, respectively, could be more lethal than the type A master variant.

DWV type B master variant dominance was, however, confirmed by Illumina sequencing (Figures 3-5). As a proportion of the total sequenced Illumina reads, DWV hits accounted for an average of $46.3 \%$ of reads in the Varroa samples and $9.7 \%$ of reads in the honey bees. The average DWV genome
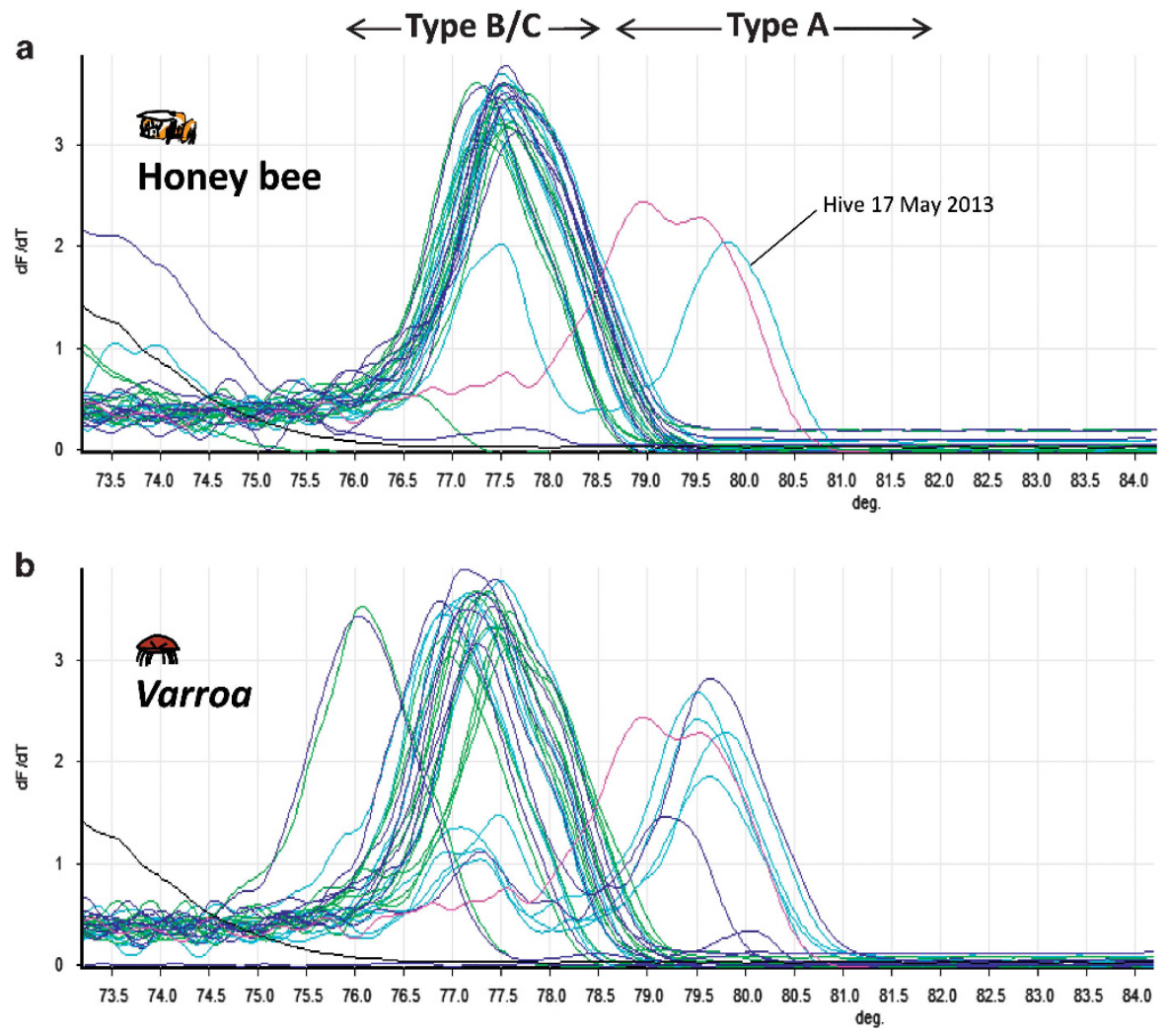

Figure 2 HRM curve analysis for DWV RdRp RT-qPCR region for three hives in the UK colonies (hive 6-blue, hive 17-cyan, hive 19green). (a) Honey bees and (b) Varroa mites distinguishing between DWV type A and B/C variants. Deformed winged symptomatic bees were used as a positive control (pink line). A no template negative control was also run (black line). 


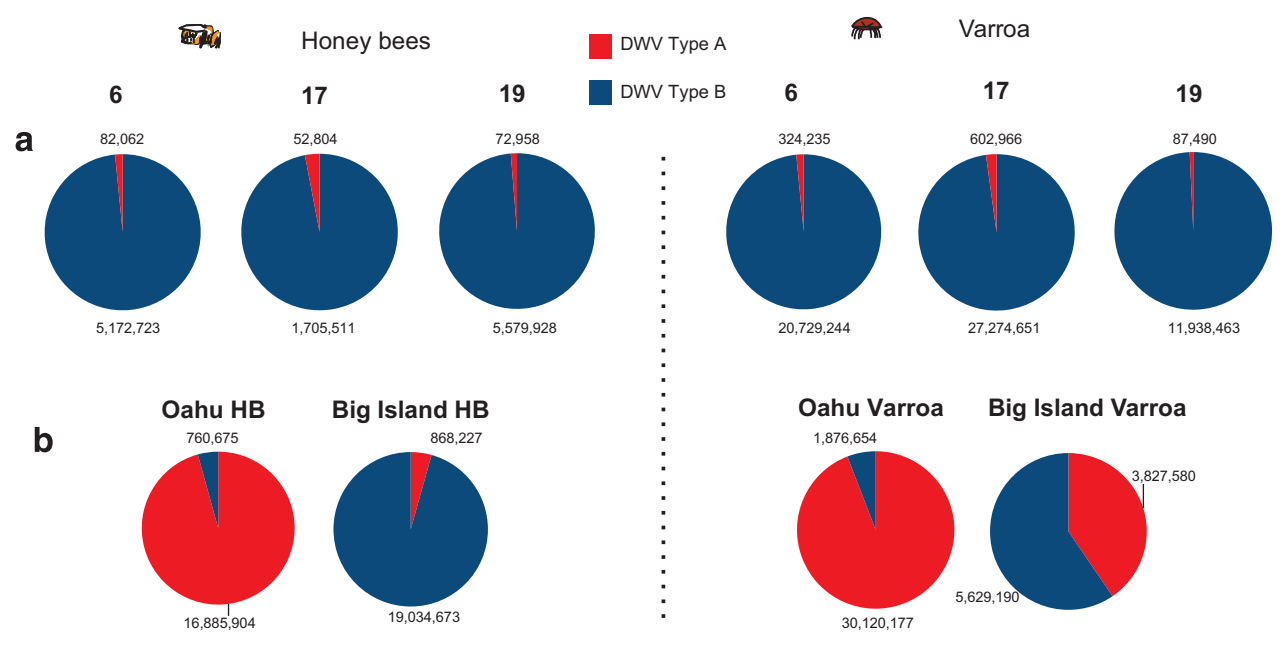

Figure 3 Proportions of DWV subgroups within colonies sequenced using Illumina Hi-seq. (a) Swindon samples collapsed into their respective hives 6, 17 and 19. (b) The Hawaiian samples from Oahu and Big Islands. A BLASTn algorithm against a custom DWV quasispecies database was used and the numbers indicate of hits to each DWV variant.

a

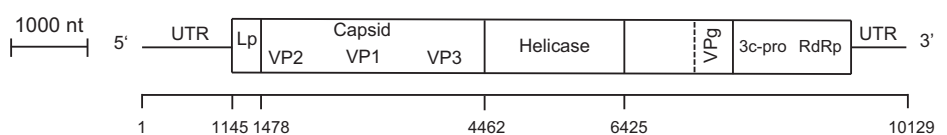

b

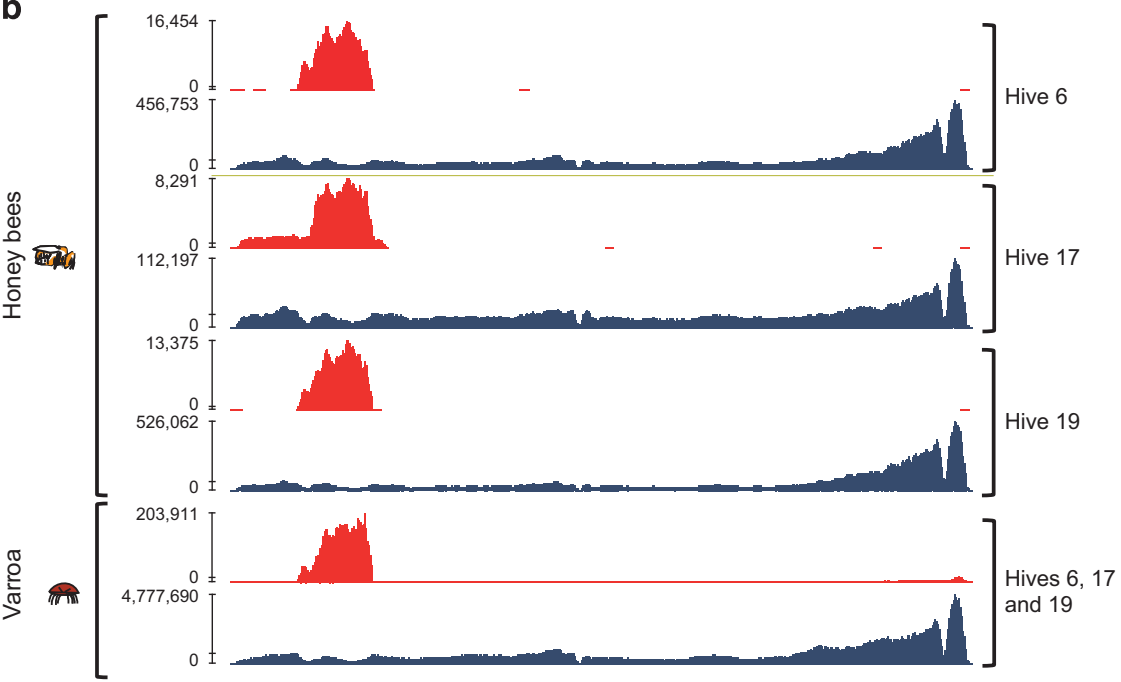

Figure 4 Genome coverage from the Illumina Hi-seq data for the Swindon colonies. (a) Map of the DWV genome adapted from Lanzi et al (2006). (b) DWV type A and B genomes (in red and blue, respectively) assembled from the Illumina NGS data from honey bees and mites from the Swindon apiary (hives 6, 17 and 19). De novo assembled VICUNA contigs that makeup these genomes for each hive were deposited in European Nucleotide Archive (ENA) under accession numbers ERS636096 to ERS636117.

coverage for the honey bee samples was $22484 X$, while the Varroa samples had an average DWV coverage of $599558 X$. VICUNA assembly produced 6410 contigs across the 18 samples (Supplementary Table S1). Sample 'Hive 6 January 2013' was used to assemble the 'Swindon' DWV type B variant (Supplementary Table S3), which was found to be $99.5 \%$ identical to the type B reference genome (VDV-1) (Supplementary Figure S1). Figure 4 also shows that the type B DWV coverage was high, with over 15 million reads aligned from the honey bee samples compared with 241000 reads aligned to the type A reference. Similarly, in the Varroa samples, 71.5 million reads aligned to type $\mathrm{B}$ compared with just over 1 million for type A. Type B reads aligned across the whole genome, whereas full genome coverage of type A was restricted to the Varroa samples. No reads unique to the Devon DWV type $\mathrm{C}$ genome could be found, whether in the honey bee or Varroa samples. In all, the honey bee- and Varroa-associated virome of the isolated UK study colonies was predominantly DWV type B (Figure 5), indicating that alternate DWV master variant competitive outcomes are possible. 


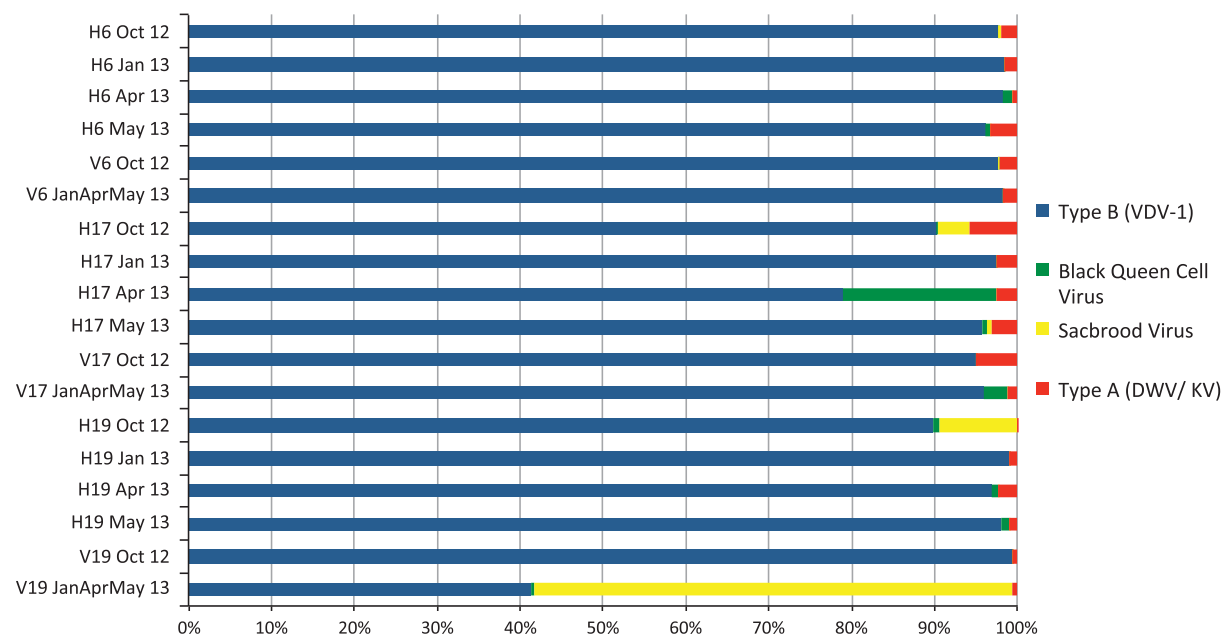

Figure 5 Virome of the Swindon apiary. Illumina reads were searched against a viral database (Figure 1) using BLASTn and the proportion of top hits associated with honey bee viruses was counted. DWV type B dominated the monthly samples in both the honey bee samples (H6, H17, H19) and the Varroa samples (V6, V17, V19).

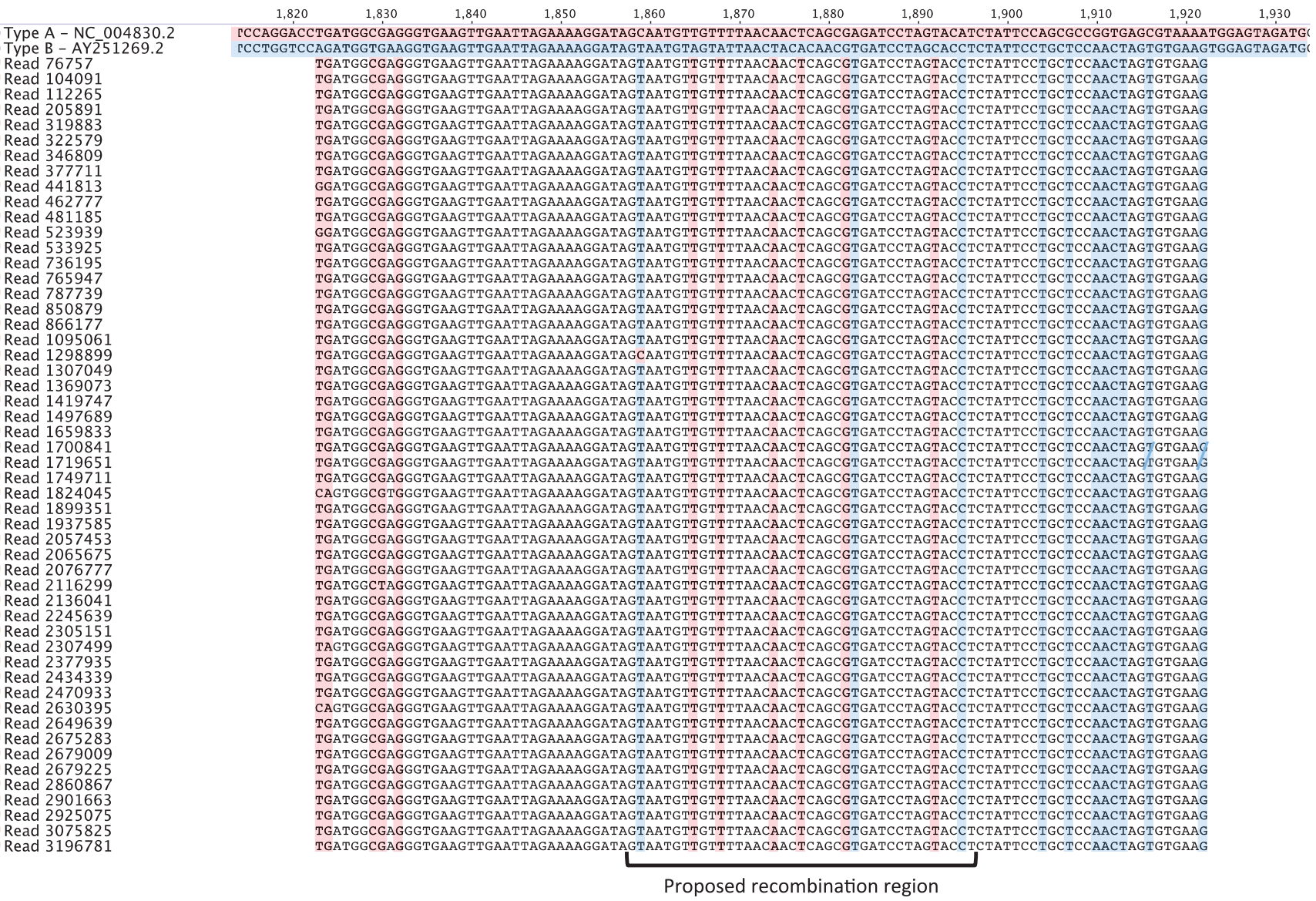

Figure 6 A multiple sequence alignment of the reads covering the recombination junction in the DWV recombinant from H19, April 2013. Output from VICUNA analysis was converted into a suitable format and imported into Geneious to visualise the reads over the type A-B recombination junction point. The DWV type A and B reference sequences are shown at the top and highlighted red and blue, respectively. Base pair substitutions common to either DWV type A and B variants are highlighted in each 100 bp Illumina read. In this example, 52 out of a total of 2464 reads is shown that covers the proposed recombination region.

De novo and reference assembly of the DWV variant genomes suggested that recombination has taken place with type A possibly being recombined out, as evidenced by the presence of DWV recombinants within the honey bee samples (Figures 4, 6 and 7). Full genome scaffolds of each recombinant were made using the VICUNA contigs. These were aligned with type $\mathrm{A}$ and $\mathrm{B}$ genomes and 


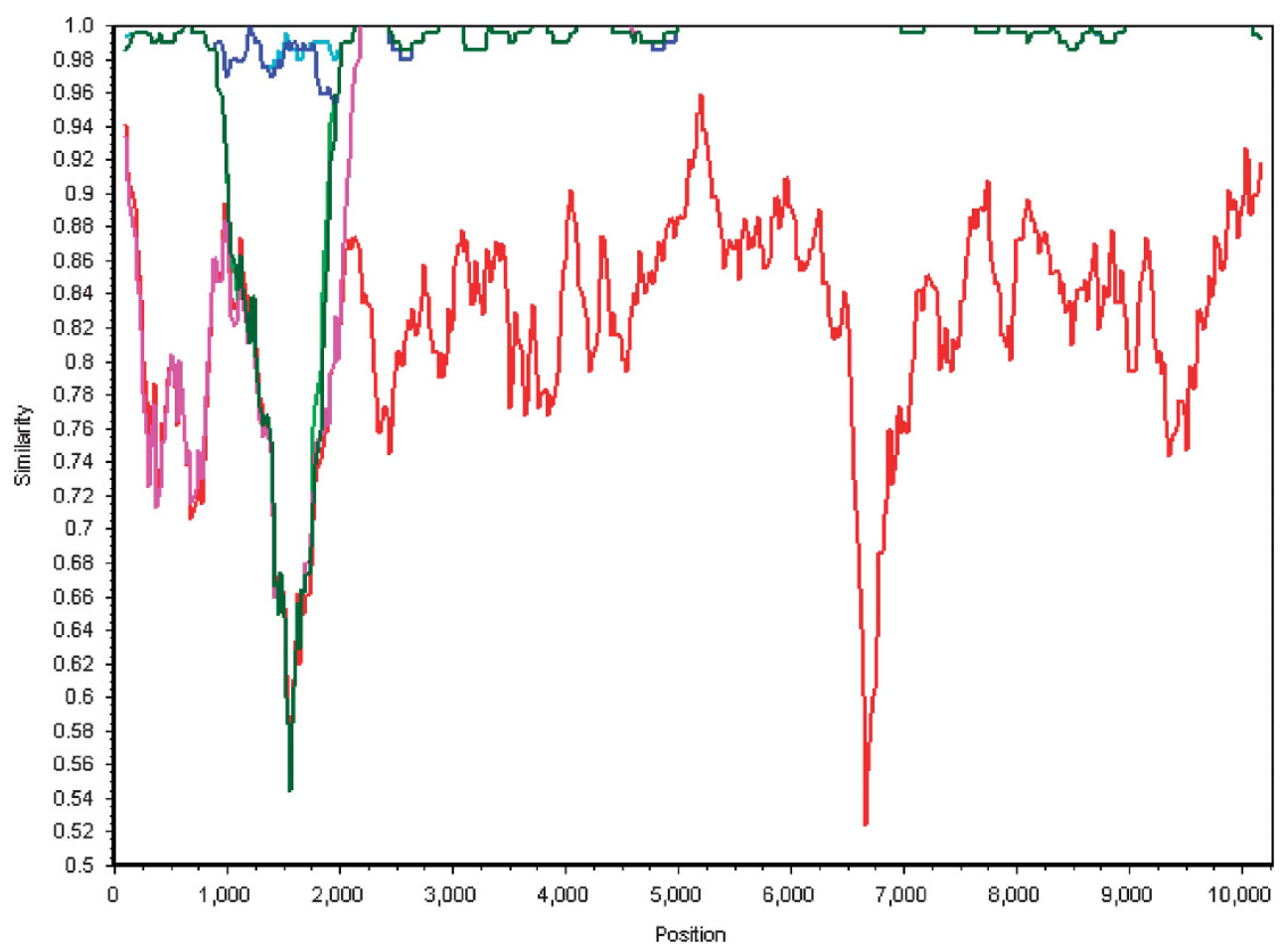

Figure 7 Simplot analyses of the different genomes present in the Swindon samples. Nucleotide similarities of various variants are compared with the type B (VDV) reference genome (AY251269.2). The type A (DWV) reference genome (NC004830.2) is shown in red. A selection of DWV genome scaffolds containing recombination in the 5' end of the genome are shown; neon and dark green (type B-A-B recombinant from January 2013 Hives 17 and 6, respectively), cyan (Swindon type B genome from Hive 6 January 2013), dark blue (Swindon type B genome from Hive 17 January 2013) and pink (type A- B recombinant, H17 April 2013). A sliding window of 200 nt was used, moving in a step of $20 \mathrm{nt}$.

Simplot (Figure 7) revealed that the recombination junction in the Swindon samples differed from that previously reported. Moore et al. (2011) showed that a recombination junction occurred in the $5^{\prime}$ untranslated region of the genome whereas the Swindon DWV type A-B recombinant junction found here occurs in the structural region of the open reading frame (Figures 4 and 7). Although full genome coverage was not achieved in both honey bee and Varroa samples by de novo assembly for type A, interestingly, reference alignment of DWV reads from the Swindon Varroa mites shows that the whole genome of type A is present at low levels (Figure 4), although HRM analysis indicated that the type A (master or any recombinants thereof) is rapidly removed in the following 5 months (see Figure 2, hive 17). A low number of type A reads (1.68\% according to BLAST analysis) present in the UK study population (Figure 3) were uniquely associated with novel recombinants (Figures 4, 6 and 7, Supplementary Table S4) in which the majority of the genome were type $\mathrm{B}$ but contained a region of type A sequence at the $5^{\prime}$ end of the genome (the UTR and leader protein, Figure 4). The number of reads within the region of recombination for each of the hives was counted to compare the depth of coverage between the two variants (Supplementary Table S4). As this is a direct comparison of the same region of the genome, that is, the $3^{\prime}$ end, which is caused by bias in reverse transcription oligo dT priming (Figure 4), the $3^{\prime}$ bias is not relevant. In all hives, the number of type $\mathrm{B}$ reads exceeded the number of type A (recombinant) reads by an order of between 4.5 (Hive 19) and 36.4 (hive 17). Therefore, the dominance of type B master variant in this UK study population appears to be correlated with a level of colony protection as it appears to exclude type A or C (and any virulent recombinants thereof).

To compare this discovery of type B dominance in this study with respect to the previous Hawaiian study (Martin et al., 2012), a small number of honey bee and Varroa Hawaiian samples with a known Varroa history were also subject to Illumina $(2 \times 100 \mathrm{bp}) \mathrm{Hi}$-seq sequencing. The same analytical VICUNA pipeline as that used for the UK samples (Figure 1) resulted in 212 contigs being assembled (Supplementary Table S1). On Oahu, where Varroa had established and caused widespread colony death, a colony analysed by Hi-seq (173 567X coverage) revealed that type $A$ dominated (Figure $3 \mathrm{~b}$ ) confirming HRM data from another 28 colonies from Oahu, which also had predominantly type A (Martin et al., 2012). However, in the colony from Big Island where Varroa had been present for less than 2 years and widespread colony collapse was yet to occur, type B dominated the sequence reads (195 760X coverage). In contrast, the Varroa sample from the 
same colony on Big Island contained a nearly equal mix of type A and B (93014X coverage), whereas Varroa from Oahu (314 713X coverage) was dominated by type A (Figure 3b). A switch in dominance between type A and B in the Big Island honey bees suggests active competition between the two DWV variants consistent with the suggested 1-3-year time lag for DWV variants adapted to mite transmission to undergo selection (Martin et al., 2012). As in Swindon, no significant matches to type C could be found in the honey bee or Varroa samples on either island. The time lag of the B to A switch in Big Island corresponds to the period when the mite becomes established but before colonies start dying. The normal outcome of this variant competition is the dominance of type A as evidenced by its transmission around the world (Berényi et al., 2007). In the Varroa-resistant Swindon apiary, once established, the avirulent type B variant appears to prevent type A from becoming dominant. Crucially, in Swindon, the Varroa mites contained a proportion of type A reads (representing the whole type A genome) which were not detected in the honey bees suggesting that effective transmission of type A from parasite to host was prevented (Figure 4).

SIE has been well documented in viruses related to DWV, for example, Tscherne et al. (2007) used cell lines to show that infection by one genotype of hepatitis $\mathrm{C}$ virus prevented infection by others. SIE best explains the phenomenon of why, despite high DWV load and Varroa infestation, the isolated UK colonies do not collapse. We speculate that coevolution of the honey bee-Varroa mite-DWV system has selected for a new stable equilibrium where both the Varroa and an avirulent type B variant of DWV protect the honey bee, and thus the colony, from the virulent type A (Figure 8). Further work to validate this and determine the mechanism of the viral exclusion is required. For example, to demonstrate whether type B can protect against type A or C at the cellular and individual honey bee-level using assays similar to those described by Ryabov et al. (2014). If true, this would be the first report of SIE acting on the Iflavirus pathogens of bees. Ironically, it may be the presence of the mite population that is protecting the colony as Varroa may be providing the opportunity for constant re-introduction of type $B$ into the population via horizontal transmission. In addition, although recombinants were present in both honey bee and Varroa samples, it is unclear whether these originate in the honey bees, Varroa or both.

It also remains unclear under what conditions type $B$ can prevail or whether similar mechanisms of protection operate in the Brazilian, USA and French populations. Although the mechanism for exclusion seen in the Swindon apiary is unclear, a unique recombinant between type $A$ and $B$ was found (Figure 6) suggesting that the full-length type A genome (Figure 4) is actively suppressed. This is the counterpart of recombination causing acute infections as described by Moore et al. (2011) and Ryabov et al. (2014). Other candidate mechanisms have previously been identified in different viruses at various stages of the viral life cycle, including blocking of virus entry to the cell at the level of receptor interaction or occupation of sites for RNA replication (Lee et al., 2005). Alternatively, the dominance of type B in the Swindon samples could be because of the induction of a differential immune response from the host such as RNAi (Hunter et al., 2010).

Studies on honey bee pathogens have suggested that natural selection favours the survival and transmission of DWV over viruses of the Acute Bee Paralysis Complex (ABPV, KBV and IAPV), which have a higher virulence (de Miranda and Genersch,

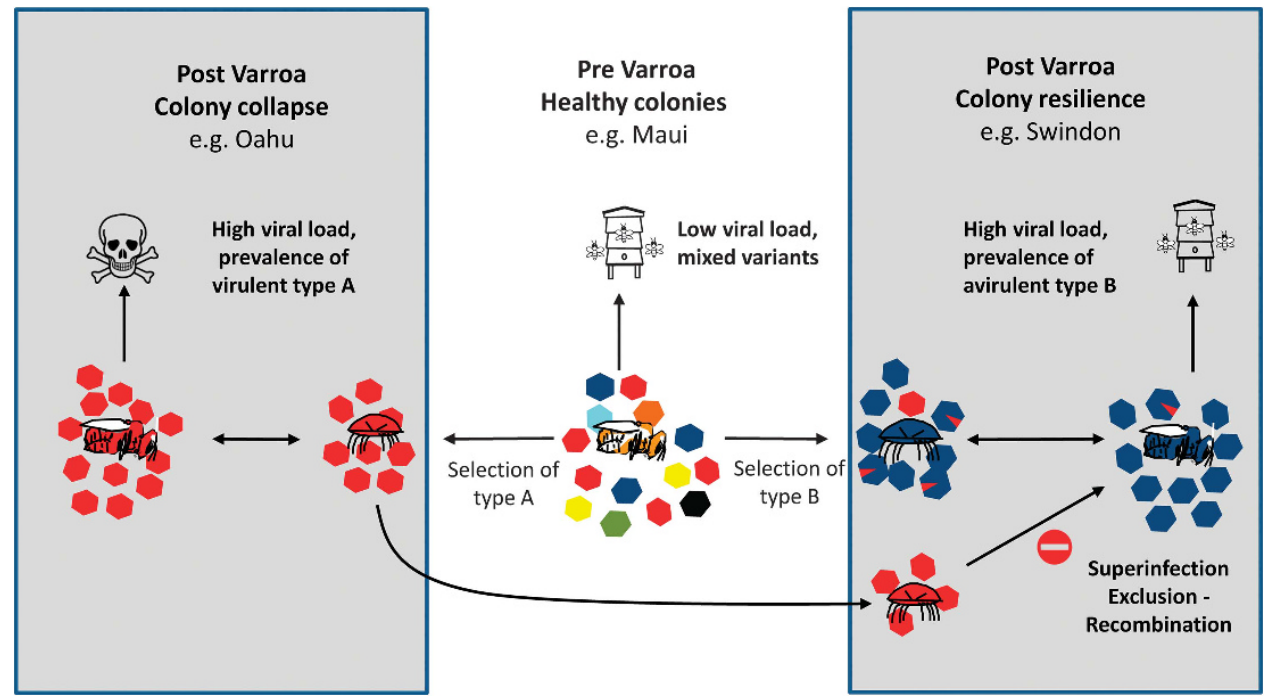

Figure 8 New honey bee-Varroa mite-DWV equilibrium. Type A DWV is represented in red and type B in blue. In Varroa-free hives, DWV exists as a cloud of variants present at low levels. In diseased hives such as Oahu, the type A is present in a Varroa-mediated transmission cycle. Whereas in Swindon, transmission of type B between bees and Varroa prevents the incursion of the type A variant into honey bees and consequently the hive survives. 
2010; Schroeder and Martin, 2012). In this scenario, virus survival requires that the pupae live long enough to enable Varroa maturation and allow onward virus transmission. For example, the acute virulence of ABPV kills both adults and pupae quickly, ending the transmission cycle as mites associated with the pupae do not survive (Schroeder and Martin, 2012). The same reasoning can be applied to the DWV quasispecies where a particular hostvariant dynamic dictates stable transmission or prevalence. Therefore, the Swindon UK population in question could have evolved to favour DWV type $B$ persistence as a result of husbandry practices that have selected for a new stable non-pathogenic equilibrium. However, this phenomenon is not peculiar to Swindon as a recent study in South Africa found only DWV type B in four study apiaries, with no type A detected in either mites or honey bees (Strauss et al., 2013). This raises the possibility that SIE may be operating on a wider scale in some geographical locations.

On the basis of our study, we hypothesise that within the swarm of DWV, owing to SIE, different viral variants are competing with two discernible outcomes. Either the disease-causing variants dominates, which can lead to colony collapse (Martin et al., 2012), or an avirulent variant can prevail, reaching high viral loads which excludes the virulent variants. In the Swindon apiary, an evolutionary stable state has been reached in which disease symptoms are minimal and colonies survive. The data show that the dominance of type B in this isolated UK apiary has been stable only over a year of sampling, but anecdotal evidence suggests that the viral makeup of the bees at the Swindon Honey bee Conservation Trust has been stable for some time longer.

The discovery of a potential SIE mechanism in honey bees gives those wishing to limit or eradicate the sources of honey bee colony decline the possibility of active intervention. For example, in the citrus industry, where SIE is used to reduce crop losses by inoculating plants with a benign variant of Citrus tristeza virus to protect against infection by a more pathogenic form (Lee and Keremane, 2013). Accordingly, the direct introduction of DWV type B could provide a form of biocontrol against further collapse of European honey bee colonies in the face of Varroa infestation.

\section{Conflict of Interest}

The authors declare no conflict of interest.

\section{Acknowledgements}

We thank the C.B. Dennis British Beekeepers' Research Trust for funding this research. GJM is funded by the British Beekeepers Association and the University of Reading. LB and SJM are funded by C. B. Dennis, Apis._M and OECD. DCS is funded by The Marine Biological
Association Senior Research Fellowship. IMJ is funded by the UK Biotechnology and Biological Sciences Research Council. We thank Ron Hoskins, Ron Hill, Eddie Eggleston and Glen Head of the Swindon Honey bee Conservation Group for their invaluable assistance in providing the bees and Varroa. We also thank Andrea C. Highfield and Joanna Schroeder for their support and helpful discussions. Finally we would like to thank the sequencing services of University of Exeter and The Genome Analysis Centre (TGAC) for their constructive suggestion. Assembled DWV contigs are available on the European Nucleotide Archive under the Study accession PRJEB8112.

\section{References}

Altschul SF, Gish W, Miller W, Myers EW, Lipman DJ. (1990). Basic local alignment search tool. J Mol Biol 215: 403-410.

Baker AC, Schroeder DC. (2008). The use of RNAdependent RNA polymerase for the taxonomic assignment of picorna-like viruses (order Picornavirales) infecting Apis mellifera L. populations. Virol J 5: 10.

Berényi O, Bakonyi T, Derakhshifar I, Köglberger H, Topolska G, Ritter W et al. (2007). Phylogenetic analysis of Deformed Wing Virus genotypes from diverse geographic origins indicates recent global distribution of the virus. Appl Environ Microbiol 73: 3605-3611.

Conte YL, Vaublanc GD, Crauser D, Jeanne F, Rousselle J-C, Bécard J-M. (2007). Honey bee colonies that have survived Varroa destructor. Apidologie 38: 566-572.

de Miranda JR, Genersch E. (2010). Deformed wing virus. J Invertebr Pathol 103(Supplement): S48-S61.

DeJong D, Soares AEE. (1997). An isolated population of Italian bees that has survived Varroa jacobsoni infestation without treatment for over 12 years. Am Bee J137: 742-745.

Di Prisco G, Zhang X, Pennacchio F, Caprio E, Li J, Evans JD et al. (2011). Dynamics of persistent and acute Deformed Wing Virus infections in honey bees, Apis mellifera. Viruses 3: 2425-2441.

Domingo E, Holland JJ. (1997). Rna virus mutations and fitness for survival. Annu Rev Microbiol 51: 151-178.

Frazer KA, Pachter L, Poliakov A, Rubin EM, Dubchak I. (2004). VISTA: computational tools for comparative genomics. Nucleic Acids Res 32: W273-W279.

Fujiyuki T, Ohka S, Takeuchi H, Ono M, Nomoto A, Kubo T. (2006). Prevalence and phylogeny of Kakugo virus, a novel insect picorna-like virus that infects the honeybee (Apis mellifera L.), under various colony conditions. J Virol 80: 11528-11538.

Gallai N, Salles J-M, Settele J, Vaissière BE. (2009). Economic valuation of the vulnerability of world agriculture confronted with pollinator decline. Ecol Econ 68: 810-821.

Highfield AC, El Nagar A, Mackinder LC, Noel LM, Hall MJ, Martin SJ et al. (2009). Deformed wing virus implicated in overwintering honeybee colony losses. Appl Environ Microbiol 75: 7212-7220.

Hunter W, Ellis J, vanEngelsdorp D, Hayes J, Westervelt D, Glick E et al. (2010). Large-scale field application of RNAi technology reducing Israeli Acute Paralysis Virus disease in honey bees (Apis mellifera, Hymenoptera: Apidae). PLoS Pathog 6: e1001160. 
Labrie SJ, Samson JE, Moineau S. (2010). Bacteriophage resistance mechanisms. Nat Rev Microbiol 8: 317-327.

Lanzi G, de Miranda JR, Boniotti MB, Cameron CE, Lavazza A, Capucci L et al. (2006). Molecular and biological characterization of Deformed Wing Virus of honeybees (Apis mellifera L.). J Virol 80: 4998-5009.

Lauring AS, Andino R. (2010). Quasispecies theory and the behavior of RNA viruses. PLoS Pathog 6: e1001005.

Lee RF, Keremane ML. (2013). Mild strain cross protection of tristeza: a review of research to protect against decline on sour orange in Florida. Front Microbiol 4: 259.

Lee Y-M, Tscherne DM, Yun S-I, Frolov I, Rice CM. (2005). Dual mechanisms of pestiviral superinfection exclusion at entry and RNA replication. J Virol 79: 3231-3242.

Lole S, Bollinger RC, Paranjape RS, Gadkari D, Kulkarni SS, Novak NG et al. (1999). Full-length human immunodeficiencey virus Type 1 genomes from subtype C-infected seroconverters in India, with evidence of intersubtype recombination. J Virol 73: 152-160.

Martin SJ, Highfield AC, Brettell L, Villalobos EM, Budge GE, Powell M et al. (2012). Global honey bee viral landscape altered by a parasitic mite. Science 336: 1304-1306.

Mondet F, de Miranda JR, Kretzschmar A, Le Conte Y, Mercer AR. (2014). On the front line: quantitative virus dynamics in honeybee (Apis mellifera L.) colonies along a new expansion front of the parasite Varroa destructor. PLoS Pathog 10: e1004323.

Moore J, Jironkin A, Chandler D, Burroughs N, Evans DJ, Ryabov EV. (2011). Recombinants between Deformed Wing Virus and Varroa destructor virus-1 may prevail in Varroa destructor-infested honeybee colonies. J Gen Virol 92: 156-161.

Mordecai GJ, Wilfert L, Martin SJ, Jones IM, Schroeder DC. (2015). Diversity in a honey bee pathogen: first report of a third master variant of the Deformed Wing Virus quasispecies. ISMEJ (in review).

Ongus JR, Peters D, Bonmatin J-M, Bengsch E, Vlak JM, van Oers MM. (2004). Complete sequence of a picorna-like virus of the genus Iflavirus replicating in the mite Varroa destructor. J Gen Virol 85: 3747-3755.

R Core Team (2015). R: A Language and Environment for Statistical Computing. $\mathrm{R}$ foundation for statistical computing: Vienna, Austria.

Ratnieks FLW, Carreck NL. (2010). Clarity on honey bee collapse? Science 327: 152-153.

Ryabov EV, Wood GR, Fannon JM, Moore JD, Bull JC, Chandler D et al. (2014). A virulent strain of deformed wing virus (DWV) of honeybees (Apis mellifera) prevails after Varroa destructor-mediated, or in vitro, transmission. PLoS Pathog 10: e1004230.

Salaman RN. (1933). Protective inoculation against a plant virus. Nature 131: 468-468.

Schroeder DC, Martin SJ. (2012). Deformed wing virus: The main suspect in unexplained honeybee deaths worldwide. Virulence 3: 589-591.

Seeley TD. (2007). Honey bees of the Arnot Forest: a population of feral colonies persisting with Varroa destructor in the northeastern United States. Apidologie 38: 19-29.

Sims D, Sudbery I, Ilott NE, Heger A, Ponting CP. (2014). Sequencing depth and coverage: key considerations in genomic analyses. Nat Rev Genet 15: 121-132.

Strauss U, Human H, Gauthier L, Crewe RM, Dietemann V, Pirk CWW. (2013). Seasonal prevalence of pathogens and parasites in the savannah honeybee (Apis mellifera scutellata). J Invertebr Pathol 114: 45-52.

Sumpter DJT, Martin SJ. (2004). The dynamics of virus epidemics in Varroa-infested honey bee colonies. J Anim Ecol 73: 51-63.

Thompson CE, Biesmeijer JC, Allnutt TR, Pietravalle S, Budge GE. (2014). Parasite pressures on feral honey bees Apis mellifera sp. PLoS ONE 9: e105164.

Tscherne DM, Evans MJ, von Hahn T, Jones CT, Stamataki Z, McKeating JA et al. (2007). Superinfection exclusion in cells infected with Hepatitis C Virus. J Virol 81: 3693-3703.

Yang X, Charlebois P, Gnerre S, Coole MG, Lennon NJ, Levin JZ et al. (2012). De novo assembly of highly diverse viral populations. BMC Genomics 13: 475.

Yue C, Genersch E. (2005). RT-PCR analysis of Deformed Wing Virus in honeybees (Apis mellifera) and mites (Varroa destructor). J Gen Virol 86: 3419-3424.

Zioni N, Soroker V, Chejanovsky N. (2011). Replication of Varroa destructor virus 1 (VDV-1) and a Varroa destructor virus 1-Deformed Wing Virus recombinant (VDV-1-DWV) in the head of the honey bee. Virology 417: 106-112.

This work is licensed under a Creative Commons Attribution 4.0 International License. The images or other third party material in this article are included in the article's Creative Commons license, unless indicated otherwise in the credit line; if the material is not included under the Creative Commons license, users will need to obtain permission from the license holder to reproduce the material. To view a copy of this license, visit http://creativecommons.org/ licenses/by/4.0/

Supplementary Information accompanies this paper on The ISME Journal website (http://www.nature.com/ismej) 